\title{
Otimização do Consumo de Energia em Redes de Rádio Heterogêneo via Cooperação
}

\author{
Roberto P. Nascimento ${ }^{1}$ \\ Programa de Computação, UFOPA, Santarém, PA \\ Stenio Fernandes ${ }^{2}$ \\ Centro de Informática, UFPE, Recife, PE \\ Djamel F. H. Sadok ${ }^{3}$
}

Centro de Informática, UFPE, Recife, PE

Resumo. O crescimento exponencial da carga de tráfego e a necessidade de acesso ubíquo provocaram uma expansão da infraestrutura de rede e uma demanda crescente de energia. Portanto, foi elaborado um modelo baseado em programação linear inteiro misto (PLIM) para otimizar a economia de energia nas redes sem fio.

Palavras-chave. Otimização de energia, Programação Linear Inteira Mista, Redes Sem Fio.

\section{Introdução}

Atualmente, as redes celulares são as mais promissoras ao atendimento de usuários móveis por conteúdo multimídia. Isto permite acesso ubíquo com altas taxas de transmissão e consumo de energia. Neste contexto, os operadores de redes de telecomunicações móveis têm de encontrar formas de atender essa nova demanda de recursos e, ao mesmo tempo, buscar formas de otimizar o consumo de energia nas RSFs. Assim, diversos estudos vêm sendo realizados com foco em estratégias eficientes para economia de energia. Chen e Wu em [1] propõem um modelo de minimização de troca de operação on/off da femtocell, onde a ociosidade desta é ampliada sempre que necessário. Os autores em [2] exploram a disponibilidade de múltiplas RANs (Radio Access Networks) propondo um framework que explora dois níveis de cooperação na rede de acesso: Cooperação intranetwork e cooperação internetwork. Ismail and Zhuang em [3] apresentam um modelo de cooperação de redes para economia de energia, baseado em um framework que permite a colaboração entre si das redes com cobertura sobrepostas.

Este trabalho, em particular, considera a investigação de cooperação celular para otimização do consumo de energia nas Redes Sem Fio (RSF) heterogêneas. O estudo foi

\footnotetext{
${ }^{1}$ roberto.nascimento@ufopa.edu.br

${ }^{2}$ sflf@cin.ufpe.br

3jamel@cin.ufpe.br
} 
realizado utilizando o modelo de Programação Linear Inteira Mista (PLIM). Na modelagem foi contemplada a existência de múltiplas RFSs e classes de serviços com custo de admissão associado. Adicionalmente, foi explorada a flutuação da carga de tráfego na rede para determinar o modo de operação (ativa/inativa) ótimo da RSF.

\section{Modelo do Sistema}

O sistema a ser analisado consiste de um conjunto $\mathcal{J}$ de Redes Sem Fio (RSF) dispostas de maneira co-localizada com diferentes tecnologias de acesso disponível (do inglês Radio Access Technology - RAT), $\mathcal{J}=\{1,2, \ldots, J\}$. Neste cenário, diversas redes sem fio (Wifi, 3G, WiMAX, etc) estão localizadas numa região geográfica formada por um conjunto de áreas de serviço, $\mathcal{N}$ com $\mathcal{N}=\{1,2, \ldots, N\}$. Como resultado, cada área de serviço $n \in \mathcal{N}$ é coberta por diferentes RSF co-localizadas, formada por macrocélulas, microcélulas e picocélulas.

Neste cenário de redes co-localizadas, a $j$-ésima RSF consiste de um enlace sem fio com $b_{j}$ recursos de rádio, os quais são compartilhados entre as requisições dos usuários. Ainda nesse cenário, tem-se vários tipos de redes de acesso, com áreas de cobertura diversificada, além de diferentes capacidades e custos. É adotado neste trabalho, que uma unidade de recursos de rádio, $b_{j}$, é o número de canais disponíveis na $j$-ésima RSF $(j \in \mathcal{J})$. Cada canal tem uma largura de banda fixa $B$.

Os usuários móveis, de posse de terminais multimodais, ao chegarem na área de serviço $n$, formado pelo ambiente de redes cooperativo, poderão ser atendidos na RSF mais adequada, de acordo com os critérios pré-estabelecidos como: custo de serviço, classe de serviço e economia de energia.

\subsection{Modelo de Otimização de Energia}

O modelo proposto explora a flutuação temporal da carga de tráfego elaborado por [3] que utiliza a flutuação de grande-escala ${ }^{1}$ (o tráfego alterna significativamente durante o período do dia) e a flutuação de pequena-escala ${ }^{2}$ (o tráfego varia ligeiramente em torno de algum valor médio).

A ótima utilização dos recursos da RSF e decisão do modo de operação, feita no momento inicial de cada período $t$, pode ser obtido usando o seguinte modelo:

$$
\begin{gathered}
\min \left\{\alpha\left[\sum_{j}^{J} \sum_{i}^{I}\left(x_{i j} \cdot c_{i j}\right)+\left(y_{j} \cdot P_{j}\right)+P_{o f f_{j}}\right]+(1-\alpha)\left[\sum_{j}^{J} \Delta P_{j}\right]\right\} \\
\text { s.a. } \sum_{i=1}^{I} x_{i j} \leq b_{j} \cdot y_{j} \quad \forall j \in \mathcal{J}
\end{gathered}
$$

\footnotetext{
${ }^{1} \mathrm{O}$ tempo é dividido em um conjunto de períodos, $\mathcal{T}=\{1,2, \ldots, T\}$, de duração constante $\tau$ horas, $T=24 / \tau$.

${ }^{2}$ Divide cada período $t(t \in \mathcal{T})$ em um conjunto de períodos menores, $\mathcal{D}=\{1,2, \ldots, D\}$, cada um com uma duração constante $\Lambda, D=\tau / \Lambda$.
} 


$$
\begin{gathered}
\sum_{j=1}^{J} x_{i j}=\rho_{i} \quad \forall i \in I \\
\sum_{j=1}^{J} y_{j} \geq 1 \\
x_{i j}>0, y_{j} \in\{0,1\}, \forall i \in I, \forall j \in \mathcal{J}
\end{gathered}
$$

A função objetivo Eq.(1) representa a minimização do consumo de energia no ambiente de redes cooperativo. As variáveis $x_{i j}$ e $y_{j}$ são variáveis de decisão que determinam o número de recursos alocados em cada RSF e o modo de operação da RSF, respectivamente.

O parâmetro $c_{i j}$ define o custo de admissão de cada classe de serviço $i$, no RAT $j$. O parâmetro $P_{j}$ e $P_{o f f_{j}}$ define o consumo de energia em cada RSF, que depende do modo de operação da RSF. O $P_{j}$ é o consumo total de energia da RSF ativa e $P_{o f f_{j}}$ é o consumo de energia de uma RSF inativa. O parâmetro $\Delta P_{j}$ determina o consumo de energia adicional necessário para ativar a BS. O parâmetro $\alpha$, com $\alpha \in(0,1)$, é um fator peso que dá uma determinada importância entre o consumo de energia e custo da troca do modo de operação da RSF, quando a RSF passar de inativa para ativa. Este parâmetro é necessário para minimizar a frequência com que o modo de operação da RSF é alternada. Assim, reduz o custo da troca do modo de operação devido o consumo de energia adicional necessário para ativar a RSF.

Na definição da função objetivo, há um trade-off entre a redução do consumo de energia (alcançada por alternar o modo de operação da RSF) com o custo de troca do modo de operação. A restrição da Eq.(2) garante que a quantidade de recursos requisitados por cada classe de serviço em cada RSF, $x_{i j}$, não ultrapasse a capacidade individual de cada RSF e total do ambiente de rede cooperativo, $\sum_{j=1}^{J} b_{j}$.

A restrição da Eq.(3) define que a quantidade de recursos alocados por cada classe de serviço a uma determinada RSF é igual a demanda do tráfego de grande-escala, $\rho_{i}$, no tempo t. O modo de operação da RSF é determinado na restrição da Eq.(4), onde o modo de operação é representado pelo binário 0 e 1 que indica a BS inativa e ativa, respectivamente. Neste ambiente de rede cooperativa há sempre, no mínimo, uma RSF ativa para garantir serviço de rede ao TM.

A restrição da Eq.(5) determina que a variável de decisão $x_{i j}$ e $y_{j}$ seja positiva e binária, respectivamente. Dessa forma, as Eqs.(1-5), determinam o ótimo modo de operação para as RSFs no ambiente de rede cooperativo; minimiza o consumo de energia na RSF durante um período de tempo $t$; determina a alocação dos recursos das RSFs baseado no tipo de tráfego e limita a frequência de troca do modo de operação das RSFs.

É possível ainda, otimizar o número de canais ativos para maximizar a economia de energia na RSF ativa. Este valor é calculado no início de cada período $d \in D$ usando o seguinte problema de otimização baseado no modelo [3]: 


$$
\max \left[\sum_{j=1}^{J} y_{j}\left(P_{j}-\left(P f_{j}+\sum_{i=1}^{I} x_{i j} \cdot P v_{j}\right)\right)\right] \quad \forall j \in \mathcal{T}
$$

onde $y_{j}$ e obtido na solução da Eqs.(1 - 5). O problema de otimização da Eq.(6) está sujeito a restrições das Eqs. $(2-3)$. O consumo de energia total $P_{j}$ é formado por dois componentes: $\mathrm{O}$ primeiro é um componente fixo que representa a fonte de alimentação da RSF e a central de refrigeração, determinado por $P f_{j}$. O segundo componente depende do número de canais ativos na RSF e conta para o amplificador de potência, feeder loss e potência transmitida, determinado por $P v_{j} . \mathrm{O} n_{j}, \operatorname{com} n_{j}=x_{i j}$, é número de canais ativos na RSF $j, j \in \mathcal{J}$. Dessa forma, o consumo de energia da RSF é:

$$
P_{j}=P f_{j}+n_{j} \cdot P v_{j}
$$

\subsection{Métricas de desempenho}

As medidas de desempenho utilizadas para avaliar o modelo proposto são:

- Economia de energia: Calculada através do framework dado nas Eqs.(1-6).

- Probabilidade de bloqueio: Das conexões da classe de serviço $i$ na RSF $j$ no tempo $t, \forall i \in I, \forall j \in \mathcal{J}, \forall t \in \mathcal{T}$, pode ser calculada utilizando a fórmula de Erlang $B$ da Eq.(8).

- Utilização: É definida como a relação entre o número total de canais e o número médio de canais ocupados. Dessa forma, é calculado a utilização dos recursos de cada RSF j, através da Eq.(9).

$$
P b_{i}=\frac{\frac{\left(\rho_{i j}\right)^{s_{j}}}{S_{j}}}{\sum_{s=1}^{S_{j}} \frac{\rho_{i j}}{s !}} \quad \forall i \in \mathcal{I} \text {. }
$$

Onde $S_{j} \leq b_{j} \cdot y_{j} . S_{j}$ determina o número de canais na RSF $j \in \mathcal{J}$.

$$
U_{j}=\frac{1}{B_{j}} \sum_{i=1}^{I} x_{i j} y_{j} \quad \forall j \in \mathcal{J} .
$$


Tabela 1: Modo de operação das RSFs

\begin{tabular}{|c||c|c|c|c|c|}
\hline Período & $1-10$ & $11-13$ & $14-18$ & 19 & $20-24$ \\
\hline \hline RSF-1 & On & On & On & On & On \\
\hline RSF-2 & On & Off & On & Off & On \\
\hline RSF-3 & Off & On & On & On & Off \\
\hline
\end{tabular}

\subsection{Parametrização do Modelo}

Para obtenção dos resultados apresentados, são considerados dois cenários com três RSFs co-localizadas, ou seja, $J=3$. No cenário 1 , o ambiente de rede analisado é formado por pico, micro e macrocélula. No cenário 2, o ambiente de rede analisado é formado por pico e microcélula.

O ambiente de rede cooperativo formado por $\mathcal{J}=\{1,2,3\}$ com a cobertura da WLAN, Celular e WiMAX indexado como 1, 2 e 3, respectivamente. É assumido que todas as RSFs iniciam no modo de operação ativo e que há três classes de serviço no sistema $I=\{1,2,3\}$ handover, nova chamada de tempo real e nova chamada de melhor esforço indexado como 1, 2 e 3, respectivamente. Os serviços de handover e tempo real são de alta prioridade, o serviço de melhor esforço tem baixa prioridade. Sendo que handover tem maior prioridade sob a classe de tempo real. Também é considerada a existência de duas áreas de serviço, $n(n=1,2 \in \mathcal{N})$.

Para o mecanismo de prioridade, é definido na RSF diferentes custos para alocação das classes de serviço, $c_{i j}$. O valor $\alpha$ determina igual importância para minimizar o consumo de energia e reduzir o custo na troca do modo de operação das RSF [3]. Os parâmetros do Cenário-1 e Cenário-2 são adaptados de [1] [3] e são apresentados a seguir: $\left\{\left\{\left[P_{1}, P_{2}, P_{3}\right]=[10 \mathrm{~W}, 200 \mathrm{~W}, 500 \mathrm{~W}]\right\},\left\{\left[P_{o f f_{1}}, P_{o f f_{2}}, P_{o f f_{3}}\right]=[2 \mathrm{~W}, 10 \mathrm{~W}, 30 \mathrm{~W}]\right\},\left\{\left[\Delta P_{1}, \Delta P_{2}, \Delta P_{3}\right]=\right.\right.$ $[1 W, 20 W, 50 W]\},\left\{\left[P f_{1}, P f_{2}, P f_{3}\right]=[6 W, 125 W, 200 W]\right\},\left\{\left[c_{11}, c_{12}, c_{13}\right]=[0.2,0.5,0.8]\right\}$, $\left.\left\{\left[c_{21}, c_{22}, c_{23}\right]=[0.4,0.4,0.7]\right\},\left\{\left[b_{1}, b_{2}, b_{3}\right]=[10,20,40]\right\},\{[\alpha, \beta, \tau, \Lambda]=[0.5,0.3,1 h, 15 \mathrm{~min}]\}\right\}$,

Contudo, no Cenário-2 o parâmetro $P_{3}=275$ e $P v_{3}=P v_{2}$ que é calculado pela Eq.7.

\section{Resultados Numéricos}

\subsection{Economia de Energia}

A Tabela 1 mostra a decisão ótima do modo de operação da RSF por diferentes períodos. O modo de operação da BS varia de acordo com a flutuação da carga de tráfego alocada em cada RSF. A RSF-1 é mantido ativo durante todo o período $t=24 \mathrm{~h}$, isso ocorre devido a rede ser do tipo picocélula, que tem como característica baixo consumo de energia, e com isso obter a utilização máxima da rede, como mostra a Fig.1(a). O modo de operação das RSFs é o mesmo para ambos os cenários apresentados.

A Tabela 2 mostra e compara a economia de energia nos dois cenários propostos através do modelo de otimização de consumo de energia, no período de $24 \mathrm{~h}$. As colunas 
Tabela 2: Economia de energia

\begin{tabular}{c||c|c||c|c|}
\multicolumn{1}{c||}{} & \multicolumn{2}{c||}{ Cenário 1 (\%) } & \multicolumn{2}{c|}{ Cenário 2 (\%) } \\
\cline { 2 - 5 } & F. Obj. 1 & F. Obj. 2 & F. Obj. 1 & F. Obj. 2 \\
\hline \hline RSF-1 & 0 & 15,83 & 0 & 6,66 \\
\hline RSF-2 & 15,83 & 24,67 & 15,83 & 26,90 \\
\hline RSF-3 & 58,75 & 64,38 & 59,37 & 65,86 \\
\hline
\end{tabular}

'F. Obj. 1' do Cenário-1 e Cenário-2 apresentam os resultados do problema de otimização de minimização do consumo de energia. Nas colunas 'F. Obj. 2' apresentam a economia de energia do problema de otimização de maximização de economia de energia quando os canais das RSFs são otimizados.

Para ambos os cenários, a RSF-1 não obteve economia de energia devido a rede permanecer ativa. Esse comportamento se deu devido ao baixo consumo de energia da rede.

Os resultados demonstram que explorar a flutuação da carga de tráfego das multiclasses de serviços, grande-escala e pequena-escala, melhora a economia de energia no ambiente de rede cooperativo para ambos os cenário apresentados. Ainda, o cenário 2 se mostrou mais eficiente em economia de energia para RSF-2.

\subsection{Utilização dos recursos de rádio e probabilidade de bloqueio}

A estratégia de economia de energia proposta passa obrigatoriamente por manter os recursos de rádio menos tempo ociosos. Para isso, adota-se as estratégias de troca do modo de operação das RSF e a otimização do número de canais ativos. Com isso, a Fig.1(a) mostra que a utilização dos recursos de rádio da RSF-1, para ambos os cenários, é alta.

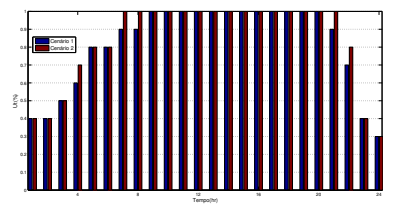

(a)

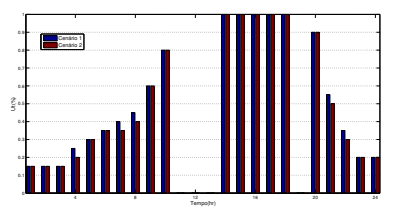

(b)

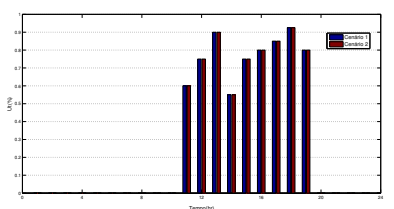

(c)

Figura 1: Utilização dos recursos de rádio: (a) RSF-1, (b) RSF-2, (c) RSF-3.

As Figs.1(b) e 1(c) mostram a utilização dos recursos de rádio da RSF-2 e RSF-3. Conforme a configuração adotada, os recurso da RSF-2 e RSF-3, quando ativos, são utilizados, de modo que os recursos sejam mantidos menos tempo ociosos.

A Fig.2(a) mostra a taxa de chegada das conexões da classe de serviço $i$ na área geográfica $n$ no período de $24 \mathrm{~h}$, baseado em [2]. 


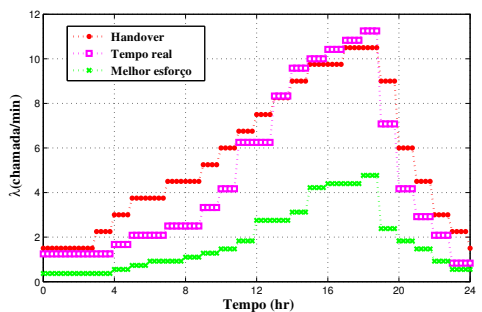

(a)

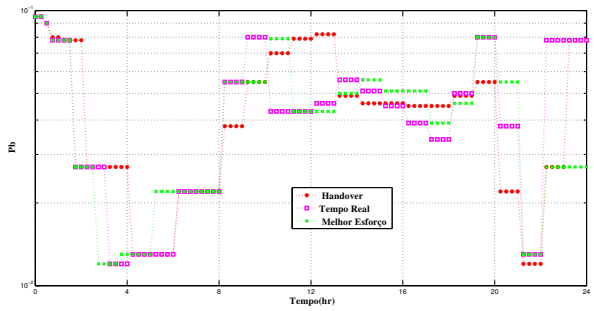

(b)

Figura 2: (a) Taxa de chegada das classes de serviço e (b) Probabilidade de bloqueio para cada classe de serviço.

A Fig.2(b) mostra a probabilidade de bloqueio de cada classe de serviço no ambiente de rede cooperativo quando todos os canais estão ativos. Como a taxa de chegada e o número de recursos são os mesmos para ambos os cenários, a probabilidade de bloqueio para ambos os cenários também é a mesma.

\section{Conclusão}

Neste artigo, foi proposto um framework para otimizar o consumo de energia nas RSFs. O sistema foi modelado para que haja controle sobre o modo de operação da RSF. Os resultados numéricos demonstram a eficiência do framework para a economia de energia e para utilização dos recursos das RSFs.

\section{Agradecimento}

Agradecemos a CAPES pelo apoio nesta pesquisa.

\section{Referências}

[1] Y. S. Chen and C. Y. Wu, A green handover protocol in two-tier OFDMA macrocellfemtocell networks, Mathematical and Computer Modelling, vol. 57, 2814-2831, (2013), DOI: 10.1016/j.mcm.2012.04.002.

[2] M. F. Hossain, K. S. Munasinghe and A. Jamalipour, Two level cooperation for energy efficiency in multi-RAN cellular network environment, IEEE Wireless Communications and Networking Conference, WCNC, 2493-2497, (2012), DOI: 10.1109/WCNC.2012.6214217.

[3] M. Ismail and W. Zhuang, Network cooperation for energy saving in green radio communications, IEEE Wireless Communications, vol. 18, 76-81, (2011), DOI:10.1109/MWC.2011.6056695. 